\title{
On a correspondence between ideals and commutativity in algebraic crossed products ${ }^{1}$
}

\author{
Johan ÖINERT and Sergei D. SILVESTROV \\ Centre for Mathematical Sciences, Lund University, Box 118, SE-221 00 Lund, Sweden \\ E-mails: johan.oinert@math.lth.se, sergei.silvestrov@math.lth.se
}

\begin{abstract}
In this paper we will give an overview of some recent results which display a connection between commutativity and the ideal structures in algebraic crossed products.
\end{abstract}

2000 MSC: 16S23, 16S35, 16W50, 16D25, 16U70

\section{Introduction}

In the recent papers $[3,4]$, we have been studying a correspondence between ideals and commutativity in algebraic crossed products. Given an algebraic crossed product $\mathcal{A}_{0} \rtimes_{\alpha}^{\sigma} G$, consider the following two statements:

S1: The coefficient ring $\mathcal{A}_{0}$ is a maximal commutative subring in $\mathcal{A}_{0} \rtimes_{\alpha}^{\sigma} G$,

S2: For every non-zero two-sided ideal $I$ in $\mathcal{A}_{0} \rtimes_{\alpha}^{\sigma} G, I \cap \mathcal{A}_{0} \neq\{0\}$.

In this paper we will give an overview of some types of crossed products for which the statements $\mathbf{S} \mathbf{1}$ and $\mathbf{S 2}$ are equivalent. We will also give an example of a situation in which these statements are not equivalent. For a general crossed product we have the following result.

Theorem $1.1([3])$. Let $\mathcal{A}=\mathcal{A}_{0} \rtimes_{\alpha}^{\sigma} G$ be an algebraic crossed product and denote the commutant of $\mathcal{A}_{0}$ by $C_{\mathcal{A}}\left(\mathcal{A}_{0}\right)=\left\{a \in \mathcal{A} \mid a b=b a, \quad \forall b \in \mathcal{A}_{0}\right\}$. If the coefficient ring $\mathcal{A}_{0}$ is commutative, then $I \cap C_{\mathcal{A}}\left(\mathcal{A}_{0}\right) \neq\{0\}$ for every non-zero two-sided ideal I in the crossed product $\mathcal{A}_{0} \rtimes_{\sigma}^{\alpha} G$.

As an immediate corollary to this theorem we get that, if $\mathcal{A}_{0}$ is assumed to be maximal commutative in $\mathcal{A}_{0} \rtimes_{\alpha}^{\sigma} G$, then $I \cap \mathcal{A}_{0} \neq\{0\}$ for every non-zero two-sided ideal $I$ in $\mathcal{A}_{0} \rtimes_{\alpha}^{\sigma} G$. Hence, in a general crossed product where the coefficient ring $\mathcal{A}_{0}$ is commutative, $\mathbf{S} 1$ always implies S2. As we will se in Section 2, S2 does not always imply $\mathbf{S 1}$.

For the convenience of the reader we shall now recall the definition and the basic properties of algebraic crossed products. For more details see e.g [2]. Throughout this article all rings are assumed to be associative rings. Given a unital ring $\mathcal{R}$ we let $U(\mathcal{R})$ denote the group of multiplication invertible elements of $\mathcal{R}$.

Definition 1.1. A $G$-crossed system is a quadruple $\left\{\mathcal{A}_{0}, G, \sigma, \alpha\right\}$, consisting of a unital ring $\mathcal{A}_{0}$, a group $G$ (with unit element $e$ ), a map $\sigma: G \rightarrow \operatorname{Aut}\left(\mathcal{A}_{0}\right)$ and a $\sigma$-cocycle map $\alpha: G \times G \rightarrow U\left(\mathcal{A}_{0}\right)$ such that for any $x, y, z \in G$ and $a \in \mathcal{A}_{0}$ the following conditions hold:

(i) $\sigma_{x}\left(\sigma_{y}(a)\right)=\alpha(x, y) \sigma_{x y}(a) \alpha(x, y)^{-1}$

(ii) $\alpha(x, y) \alpha(x y, z)=\sigma_{x}(\alpha(y, z)) \alpha(x, y z)$

(iii) $\alpha(x, e)=\alpha(e, x)=1_{\mathcal{A}_{0}}$

\footnotetext{
${ }^{1}$ Presented at the $3^{\text {rd }}$ Baltic-Nordic Workshop "Algebra, Geometry, and Mathematical Physics", Göteborg, Sweden, October 11-13, 2007.
} 
Let $\left\{u_{s}\right\}_{s \in G}$ be a copy (as a set) of $G$. Given a $G$-crossed system $\left\{\mathcal{A}_{0}, G, \sigma, \alpha\right\}$, we denote by $\mathcal{A}_{0} \rtimes_{\alpha}^{\sigma} G$ the free left $\mathcal{A}_{0}$-module having $\left\{u_{s}\right\}_{s \in G}$ as its basis and we define a multiplication on this set by

$$
\left(a_{1} u_{x}\right)\left(a_{2} u_{y}\right)=a_{1} \sigma_{x}\left(a_{2}\right) \alpha(x, y) u_{x y}
$$

for all $a_{1}, a_{2} \in \mathcal{A}_{0}$ and $x, y \in G$ and extend it bilinearly to all of $\mathcal{A}_{0} \rtimes_{\alpha}^{\sigma} G$. Each element of $\mathcal{A}_{0} \rtimes_{\alpha}^{\sigma} G$ may be expressed as a formal sum $\sum_{g \in G} a_{g} u_{g}$ where $a_{g} \in \mathcal{A}_{0}$ and $a_{g}=0_{\mathcal{A}_{0}}$ for all but a finite number of $g \in G$. Explicitly, the addition and multiplication of two arbitrary elements $\sum_{s \in G} a_{s} u_{s}, \sum_{t \in G} b_{t} u_{t} \in \mathcal{A}_{0} \rtimes_{\alpha}^{\sigma} G$ is given by

$$
\begin{aligned}
\sum_{s \in G} a_{s} u_{s}+\sum_{t \in G} b_{t} u_{t} & =\sum_{g \in G}\left(a_{g}+b_{g}\right) u_{g} \\
\left(\sum_{s \in G} a_{s} u_{s}\right)\left(\sum_{t \in G} b_{t} u_{t}\right) & =\sum_{(s, t) \in G \times G}\left(a_{s} u_{s}\right)\left(b_{t} u_{t}\right)=\sum_{(s, t) \in G \times G} a_{s} \sigma_{s}\left(b_{t}\right) \alpha(s, t) u_{s t} \\
& =\sum_{g \in G}\left(\sum_{\substack{\{(s, t) \in G \times G \mid \\
s t=g\}}} a_{s} \sigma_{s}\left(b_{t}\right) \alpha(s, t)\right) u_{g}
\end{aligned}
$$

Proposition $1.1([2])$. Let $\left\{\mathcal{A}_{0}, G, \sigma, \alpha\right\}$ be a $G$-crossed system. Then $\mathcal{A}_{0} \rtimes_{\alpha}^{\sigma} G$ is an associative unital ring (with the multiplication defined in (1.1)).

Definition 1.2. The ring $\mathcal{A}_{0} \rtimes_{\alpha}^{\sigma} G$ is called the crossed product of the $G$-crossed system $\left\{\mathcal{A}_{0}, G, \sigma, \alpha\right\}$.

The coefficient ring $\mathcal{A}_{0}$ is naturally embedded as a subring into $\mathcal{A}_{0} \rtimes_{\alpha}^{\sigma} G$ via the canonical isomorphism $\iota: \mathcal{A}_{0} \hookrightarrow \mathcal{A}_{0} \rtimes_{\alpha}^{\sigma} G$ defined by $a \mapsto a u_{e}$. Instead of $\iota\left(\mathcal{A}_{0}\right)$ we will simply write $\mathcal{A}_{0}$.

Remark 1.1. If $k$ is a field and $\mathcal{A}$ is a $k$-algebra, then so is $\mathcal{A}_{0} \rtimes_{\alpha}^{\sigma} G$.

Depending on the nature of the maps $\sigma$ and $\alpha$ we will give different names to the crossed product $\mathcal{A}_{0} \rtimes_{\alpha}^{\sigma} G$. If the map $\alpha$ is trivial, i.e $\alpha(x, y)=1_{\mathcal{A}_{0}}$ for every $(x, y) \in G \times G$, then we shall write $\mathcal{A}_{0} \rtimes^{\sigma} G$ and refer to it as a skew group ring. If, on the other hand, $\sigma$ is trivial, i.e. $\sigma_{g}=\operatorname{id}_{\mathcal{A}_{0}}$ for every $g \in G$, then we shall write $\mathcal{A}_{0} \rtimes_{\alpha} G$ and refer to it as a twisted group ring. A crossed product where both of the maps $\sigma$ and $\alpha$ are trivial is written as $\mathcal{A}_{0} \rtimes G$ and is simply refered to as a group ring.

\section{Group rings, skew group rings and twisted group rings}

If one wants to talk about maximal commutativity of $\mathcal{A}_{0}$, it does not really make sense unless we assume that $\mathcal{A}_{0}$ is commutative itself, so from now on we will only consider crossed products where $\mathcal{A}_{0}$ is commutative. Note that if the group $G$ is trivial, i.e. $G=\{e\}$, then $\mathbf{S} \mathbf{1}$ and $\mathbf{S 2}$ are always true. In the further discussion we will therefore assume that $G \neq\{e\}$. We will continue the further investigation by breaking it down into special cases of crossed products.

Example 2.1 (group rings). Let $\mathcal{A}_{0}$ be a unital ring and $G$ any (non-trivial) group and denote the group ring by $\mathcal{A}_{0} \rtimes G$. Note that this corresponds to the crossed product with trivial $\sigma$ and $\alpha$ maps. We may define the so called augmentation map $\epsilon: \mathcal{A}_{0} \rtimes G \rightarrow \mathcal{A}_{0}, \sum_{s \in G} a_{s} u_{s} \mapsto \sum_{s \in G} a_{s}$, and it is straightforward to check that it is in fact a ring morphism. The kernel of this map, $\operatorname{ker}(\epsilon)$ is a two-sided ideal in $\mathcal{A}_{0} \rtimes G$ and it is not hard to see that $\operatorname{ker}(\epsilon) \cap \mathcal{A}_{0}=\{0\}$. This gives us an example of a non-zero two-sided ideal which has zero intersection with the coefficient ring 
$\mathcal{A}_{0}$, i.e. S2 is false. However, for each $s \in G, u_{s}$ commutes with every element in $\mathcal{A}_{0}$ and hence $\mathbf{S 1}$ is never true for a group ring (when $G \neq\{e\}$ ). In other words, in a group ring the two statements $\mathbf{S} \mathbf{1}$ and $\mathbf{S} \mathbf{2}$ are always equivalent.

For skew group rings we have the following theorems.

Theorem 2.1 ([4]). If $\mathcal{A}_{0} \rtimes^{\sigma} G$ is a skew group ring where the coefficient ring $\mathcal{A}_{0}$ is an integral domain and the group $G$ is abelian, then the two assertions $\mathbf{S} 1$ and $\mathbf{S} 2$ are equivalent.

Theorem 2.2 ([4]). If $\mathcal{A}_{0} \rtimes^{\sigma} G$ is a skew group ring where the coefficient ring $\mathcal{A}_{0}$ is commutative and $G$ is a torsion-free abelian group, then the two assertions $\mathbf{S 1}$ and $\mathbf{S 2}$ are equivalent.

Remark 2.1. Note that in the previous theorems, the action $\sigma$ can be trivial, but in that case the situation is already described by Example 2.1.

Example 2.2 (the algebra associated to a dynamical system). In $[5,6,7]$ the authors studies crossed product algebras associated to dynamical systems. Suppose that we are given a nonempty set $X$ and a bijection $\sigma: X \rightarrow X$. Then $(X, \sigma)$ is a discrete dynamical system where the action of $n \in \mathbb{Z}$ on $x \in X$ is given by $n \mapsto \sigma^{\circ(n)}(x)$. By $\mathbb{C}^{X}$ we denote the algebra of functions $X \rightarrow \mathbb{C}$ under the usual pointwise operations of addition and multiplication. If we are given a subalgebra $A \subseteq \mathbb{C}^{X}$ such that it is invariant under $\sigma$ and $\sigma^{-1}$, i.e. such that if $h \in A$ then $h \circ \sigma \in A$ and $h \circ \sigma^{-1} \in A$, then $\sigma$ induces an automorphism $\tilde{\sigma}: A \rightarrow A$ defined by $\tilde{\sigma}(f)=f \circ \sigma$ by which $\mathbb{Z}$ acts on $A$ via iterations. We may now define the skew group algebra $A \rtimes_{\tilde{\sigma}} \mathbb{Z}$.

In the current situation the coefficient algebra $A$ is commutative and the group $(\mathbb{Z},+)$ is clearly torsion-free and abelian, hence Theorem 2.2 is applicable. We may conclude that Theorem 2.2 is a generalization of certain parts of Corollary 4.5 in [6] and Theorem 4.5, Theorem 4.6, Corollary 4.7, Theorem 6.2 in [7].

In a twisted group ring $\mathcal{A}_{0} \rtimes_{\alpha} G$, just like for group rings mentioned above, the action $\sigma$ is trivial and hence for each $s \in G$ the element $u_{s}$ commutes with every element in $\mathcal{A}_{0}$. In other words, $\mathcal{A}_{0}$ is never maximal commutative in a twisted group ring (when $G \neq\{e\}$ ).

Example 2.3 (the field of complex numbers). Let $\mathcal{A}_{0}=\mathbb{R}, G=\left(\mathbb{Z}_{2},+\right)$ and define the cocycle $\alpha: \mathbb{Z}_{2} \times \mathbb{Z}_{2} \rightarrow \mathbb{R} \backslash\{0\}$ by $\alpha(\overline{0}, \overline{0})=1, \alpha(\overline{0}, \overline{1})=1, \alpha(\overline{1}, \overline{0})=1$ and $\alpha(\overline{1}, \overline{1})=-1$. It is easy to see that $\mathbb{R} \rtimes_{\alpha} \mathbb{Z}_{2} \cong \mathbb{C}$. Clearly this twisted group ring is a field and hence simple. Therefore, $\mathbb{C}$ is the only non-zero ideal and clearly $\mathbb{C} \cap \mathbb{R} \neq\{0\}$. However, as has already been mentioned, the coefficient ring $\mathbb{R}$ is not maximal commutative in $\mathbb{C}$. Example 2.3 shows that in a twisted group ring, $\mathbf{S} 1$ may be false even though $\mathbf{S 2}$ is true.

\section{General crossed products}

A crossed product $\mathcal{A}_{0} \rtimes_{\alpha}^{\sigma} G$, where neither of the maps $\sigma$ and $\alpha$ are trivial and hence not treated in the previous section, will be refered to as a general crossed product. For this type of crossed products we are not able to say as much as we would want to.

Theorem 3.1. If $\mathcal{A}_{0} \rtimes_{\alpha}^{\sigma} G$ is a crossed product where $\mathcal{A}_{0}$ is an integral domain, $G$ is an abelian torsion-free group and $\alpha$ is such that $\alpha(s, t)=1_{\mathcal{A}_{0}}$ whenever $\sigma_{s}=\operatorname{id}_{\mathcal{A}_{0}}$ or $\sigma_{t}=\operatorname{id}_{\mathcal{A}_{0}}$, then the two assertions $\mathbf{S} 1$ and $\mathbf{S 2}$ are equivalent.

Proof. It is clear from Theorem 1.1 that $\mathbf{S 1} \Longrightarrow \mathbf{S 2}$. Suppose that $\mathcal{A}_{0}$ is not maximal commutative. Since $\mathcal{A}_{0}$ is an integral domain, this means that there exists some $s \in G \backslash\{e\}$ such 
that $\sigma_{s}=\operatorname{id}_{\mathcal{A}_{0}}$. For arbitrary $g, h \in G$ we may use condition (ii) in Definition 1.1 and the assumptions we made on $\alpha$ to arrive at

$$
\underbrace{\alpha(s, g)}_{=1_{\mathcal{A}_{0}}} \alpha(s g, h)=\underbrace{\sigma_{s}(\alpha(g, h))}_{=\alpha(g, h)} \underbrace{\alpha(s, g h)}_{=1_{\mathcal{A}_{0}}}
$$

and since $G$ is abelian we get $\alpha(g s, h)=\alpha(s g, h)=\alpha(g, h)$. Let I be the two-sided ideal generated by $1_{\mathcal{A}_{0}}+u_{s}$, which is an element that commutes with all of $\mathcal{A}_{0}$. The ideal $I$ is obviously non-zero and furthermore, it is spanned by elements of the form $a_{g} u_{g}\left(1_{\mathcal{A}_{0}}+u_{s}\right) a_{h} u_{h}$ where $g, h \in G$ and $a_{g}, a_{h} \in \mathcal{A}_{0}$. We may now rewrite this expression.

$$
\begin{aligned}
a_{g} u_{g}\left(1_{\mathcal{A}_{0}}+u_{s}\right) a_{h} u_{h} & =a_{g} u_{g} a_{h}\left(1_{\mathcal{A}_{0}}+u_{s}\right) u_{h} \\
& =a_{g} u_{g} a_{h} u_{h}+a_{g} u_{g} a_{h} u_{s} u_{h} \\
& =a_{g} \sigma_{g}\left(a_{h}\right) u_{g} u_{h}+a_{g} \sigma_{g}\left(a_{h}\right) u_{g} u_{s} u_{h} \\
& =a_{g} \sigma_{g}\left(a_{h}\right) \alpha(g, h) u_{g h}+a_{g} \sigma_{g}\left(a_{h}\right) \underbrace{\alpha(g, s)}_{1_{\mathcal{A}_{0}}} u_{g s} u_{h} \\
& =\underbrace{a_{g} \sigma_{g}\left(a_{h}\right) \alpha(g, h)}_{:=b} u_{g h}+a_{g} \sigma_{g}\left(a_{h}\right) \underbrace{\alpha(g s, h)}_{=\alpha(g, h)} u_{g s h} \\
& =b u_{g h}+b u_{g s h}
\end{aligned}
$$

Since $G$ is abelian, it is clear that any element of $I$ may be written in the form

$$
\sum_{t \in G}\left(c_{t} u_{t}+c_{t} u_{t s}\right)
$$

for some $c_{t} \in \mathcal{A}_{0}$, where $t$ only runs over a finite subset of $G$. By assumtion $s \neq e$ and hence $t \neq t s$ for every $t \in G$. In particular this means that every contribution from $c_{e}$ to the $e$-graded part of the element in (3.1) comes with an equal contribution to the $s$-graded part. Similarly $c_{s}: \mathrm{s}$ contribution to the $s$-graded part equals its contribution to the $c_{s^{2}}$-graded part. Furthermore, $G$ is assumed to be torsion-free, i.e. $s^{n} \neq e$ for every $n \in \mathbb{Z} \backslash\{0\}$, and hence the element in (3.1) can never be a non-zero element of degree $e$, which means $I \cap \mathcal{A}_{0}=\{0\}$. By contra positivity we conclude that $\mathbf{S} 2 \Longrightarrow \mathbf{S} 1$ and this finishes the proof.

Remark 3.1. Note that, a twisted group ring can never fit into the conditions of Theorem 3.1, because if $\sigma$ is trivial, then the conditions force $\alpha$ to be trivial as well.

Finite groups are clearly not torsion-free, but Example 3.1 gives an example of a situation where $\mathbf{S 1}$ and $\mathbf{S 2}$ are in fact equivalent for a general crossed product graded by a finite group. This raises the question whether or not Theorem 3.1 can be generalized to general crossed products graded by more general groups.

Example 3.1 (central simple algebras). Let $A$ be a finite-dimensional central simple algebra over a field $F$. By Wedderburn's theorem $A \cong M_{i}(D)$ where $D$ is a division algebra over $F$ and $i$ is some integer. If $K$ is a maximal separable subfield of $D$ then $[K: F]=n$ where $[D: F]=n^{2}$. We shall assume that $K$ is normal over $F$ and that $[A: F]=[K: F]^{2}$ (see [1] for motivation). Let $\operatorname{Gal}(K / F)$ be the Galois group of $K$ over $F$. For $k \in K$ and $\sigma_{s} \in \operatorname{Gal}(K / F)$ we shall write $\sigma_{s}(k)$ for the image of $k$ under $\sigma_{s}$. By the Noether-Skolem theorem there is an invertible element $u_{s} \in A$ such that $\sigma_{s}(k)=u_{s} k u_{s}^{-1}$ for every $k \in K$. One can show that the $u_{s}$ 's are linearly independent over $K$. However, the linear span over $K$ of the $u_{s}$ 's has dimension $n^{2}$ over $F$, hence must be all of $A$. In short $A=\left\{\sum_{s \in G} k_{s} u_{s} \mid k_{s} \in K\right\}$. If $\sigma_{s}, \sigma_{t} \in \operatorname{Gal}(K / F)$ and $k \in K$, then $u_{s} u_{t} k u_{t}^{-1} u_{s}^{-1}=u_{s} \sigma_{t}(k) u_{s}^{-1}=\sigma_{s t}(k)=u_{s t} k u_{s t}^{-1}$. This says that $u_{s t}^{-1}\left(u_{s} u_{t}\right) \in C_{A}(K)=K$, in other words $u_{s} u_{t}=f(s, t) u_{s t}$ where $f(s, t) \neq 0$ is in $K$. Since $A$ is an associative algebra one 
may verify that $f: \operatorname{Gal}(K / F) \times \operatorname{Gal}(K / F) \rightarrow K \backslash\{0\}$ is in fact a cocycle. By Theorem 4.4.1 in [1], if $K$ is a normal extension of $F$ with Galois group $\operatorname{Gal}(K / F)$ and $f$ is a cocycle (factor set), then the crossed product $K \rtimes_{f}^{\sigma} \operatorname{Gal}(K / F)$ is a central simple algebra over $F$ and hence in this situation both $\mathbf{S 1}$ and $\mathbf{S 2}$ are in fact true.

\section{Acknowledgement}

We are grateful to Freddy Van Oystaeyen for useful discussions on the topic of this article. This work was supported by the Swedish Foundation of International Cooperation in Research and Higher Education (STINT), the Crafoord Foundation, The Royal Physiographic Society in Lund, The Swedish Royal Academy of Sciences and "LieGrits", a Marie Curie Research Training Network funded by the European Community as project MRTN-CT 2003-505078.

\section{References}

[1] I. N. Herstein. Noncommutative Rings. The Carus Mathematical Monographs, No. 15, The Math. Assoc. of America, 1968.

[2] C. Năstăsescu and F. Van Oystaeyen. Methods of Graded Rings. Lecture Notes in Math. 1836, Springer-Verlag, Berlin, 2004.

[3] J. Öinert and S.D. Silvestrov. Commutativity and ideals in algebraic crossed products. J. Gen. Lie Theory Appl. 2 (2008) (to be published).

[4] J. Öinert and S.D. Silvestrov. Ideals in crossed products and skew group rings. In preparation.

[5] C. Svensson, S. Silvestrov, and M. de Jeu. Dynamical sytems and commutants in crossed products. Int. J. Math. 18 (2007), 455-471.

[6] C. Svensson, S. Silvestrov, and M. de Jeu. Connections between dynamical systems and crossed products of Banach algebras by $\mathbb{Z}$. In procceding "Operator Theory, Analysis and Mathematical Physics", OTAMP-2006, June 15-22, 2006, Lund, Sweden (to be published); arXiv: math/0702118v3 (math.DS).

[7] C. Svensson, S. Silvestrov, and M. de Jeu. Dynamical systems associated with crossed products. In "Operator Methods in Fractal Analysis, Wavelets and Dynamical Systems" (to be published), Preprint arXiv:0707.1881v2 (math.0A). 